\title{
Puncture of thoracic lesions under sonographic guidance
}

\author{
M AFSCHRIFT, P NACHTEGAELE, D VOET, L NOENS, W VAN HOVE, M VAN DER \\ STRAETEN, G VERDONK
}

From the Departments of Internal Medicine, Echography, Endocrinology, Haematology, and Pneumology, Akademisch Ziekenhuis, Ghent, Belgium

\begin{abstract}
Thirty-six punctures of thoracic lesions have been performed with a compound B-scanner or a real-time linear-array scanner for guidance. Twenty-three fluid collections were punctured and aspiration biopsies were performed on 13 echogenic lesions. All the punctures were successful at the first attempt. No complications occurred. The results confirm the usefulness of sonography for guiding punctures of thoracic fluid effusions and solid masses. Usually a static B-scanner is sufficient, but when masses are small or surrounded by vital structures puncture may be controlled by a real-time scanner.
\end{abstract}

Ultrasound has been used successfully to determine the presence of fluid collections in the pleural and pericardial spaces and of solid masses originating from the lung, mediastinum, and chest wall. ${ }^{1-13} \mathrm{But}$, as the aerated lung reflects all the ultrasonic energy, thoracic lesions can be detected only if they are in close contact with the chest wall. When an abnormal region can be visualised sonographically, it may easily be punctured under sonographic control. This report describes percutaneous needle puncture of various thoracic fluid collections and masses under ultrasonic guidance.

\section{Material and methods}

Thirty-six punctures were performed in 31 patients. Twenty-three fluid collections were aspirated and aspiration biopsies were done on 1.3 solid lesions. The 23 fluid collections included 18 pleural effusions (fig 1), four pericardial effusions and one thoracic meningomyelocele. The effusions were of inflammatory origin except in two cases of pericarditis. Among the solid lesions there were four malignant thymomas (fig 2), two lymphomas, one malignant Schwannoma, three lung tumours, one leukaemic pulmonary infiltration, one pleural metastasis without pleural effusion (fig 3), and one thickened pleura.

The thickness of the thoracic lesions at the site of the puncture ranged from 1 to $7 \mathrm{~cm}$. If it exceeded 3

Address for reprint requests: Professor Dr G Verdonk, Departments of Internal Medicine and Echography, Akademisch Ziekenhuis, De Pintelaan 135, B-9000 Ghent, Belgium. cm the punctures were guided by a static B-scanner (Picker Echoview System 80; probe $2.25 \mathrm{MHz}$ ). With this method the puncture site is determined after careful scanning of the entire abnormal region. The depth of the puncture is measured on the screen and the direction of the needle is determined by the position of the transducer on the skin. When the abnormal area was small, punctures were done through the central canal of a real-time transducer (5.0 MHz ADR-Kranzbühler linear-array scanner). With this method the area to be punctured is under constant visual control during the procedure. The depth of the puncture was again measured on the screen. The tip of the penetrating needle could often be visualised during the procedure. In this series 25

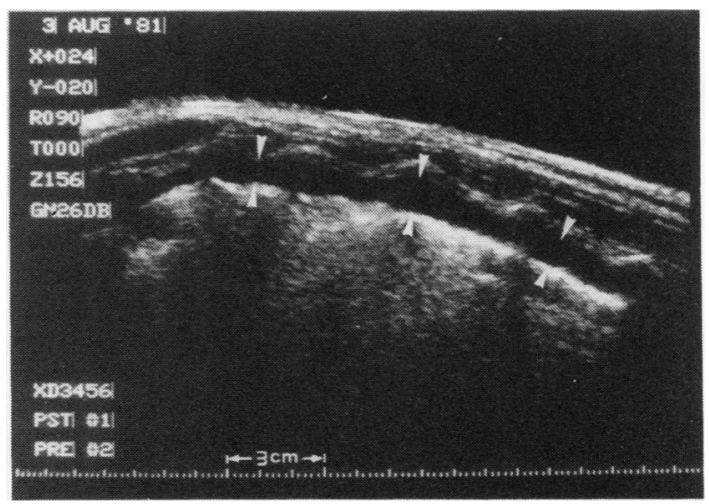

Fig 1 Parasagittal sonogram: echo-free area between the chest wall and the lung: pleural effusion (arrow-heads). 


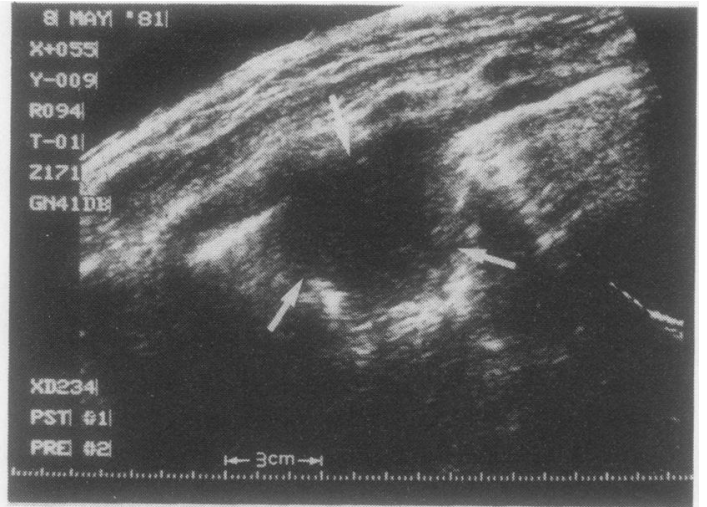

Fig 2 Parasagittal cross-section to the right of the sternum showing echogenic mass, with irregular shape and non-homogenous echo pattern: thymoma (arrows).

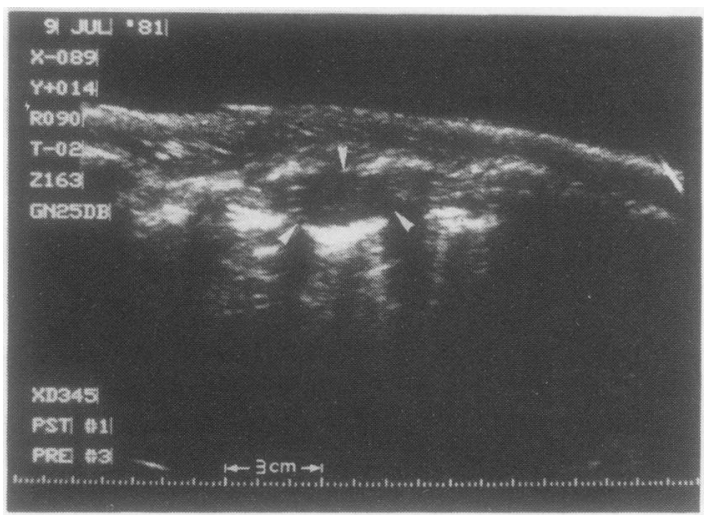

Fig 3 Cross-section through a small echogenic pleural nodule: metastasis (arrow-heads). punctures were guided by static scanning, 11 by real-time scanning.

Of the 18 pleural effusions, 16 were punctured with the help of a static B-scanner and two under real-time sonographic guidance. Four pericardial effusions were punctured, two at the left sternal border, one at the cardiac apex, and one at the right sternal border. The last two were guided by a realtime scanner. One meningomyelocele presented as a large mass at the left pulmonary apex and was easily punctured with static B-scan guidance. Fine-needle aspirations were performed in 13 solid masses, seven of them being guided by real-time scanning.

\section{Results}

In this series all the punctures were successful at the first attempt. Among the fluid collections, five were loculated and contained pus. In two cases of pericarditis malignant cells were seen in the aspirated fluid; malignant cells were also detected in the aspirated material of all 12 solid tumours (table). No malignant cells were aspirated from the thickened pleura.

No complications occurred during or after the punctures. There were no cases of pneumothorax and no signs of bleeding.

\section{Discussion}

Punctures of pleural effusions are most often performed after clinical or fluoroscopic localisation. With these methods, however, neither the accurate location of the diaphragm nor the thickness of the pleural effusion at a particular puncture site is known. Ultrasonography permits easy localisation of even small pleural effusions. The depth at which

Positive cytological fundings after echo-guided aspiration

\begin{tabular}{lllll}
\hline $\begin{array}{l}\text { Patient } \\
\text { No }\end{array}$ & Sex & Age $(y)$ & Initial diagnosis & Cytological diagnosis \\
\hline 1 & F & 48 & Chronic leukemia, pericarditis & Leukaemic pericarditis \\
2 & M & 59 & Bronchial carcinoma, pericarditis & Malignant pericardial effusion \\
3 & M & 24 & Mediastinal tumour & Malignant lymphoma with Steinberg-like cells \\
4 & M & 23 & Mediastinal tumour & Non-Hodgkin lymphoma or malignant thymoma \\
5 & F & 40 & Mediastinal tumour* & Non-Hodgkin lymphoma \\
6 & M & 23 & Mediastinal tumour & Non-Hodgkin lymphoma or malignant thymoma \\
7 & M & 27 & Mediastinal tumour* & Malignant thymoma \\
8 & M & 20 & Myelosclerosis, later ANLL; peripheral & Leukaemic lung infiltration \\
9 & M & 52 & lung infiltration & Poorly differentiated carcinoma \\
10 & M & 78 & Peripheral lung infiltration & Squamous-cell carcinoma \\
11 & F & 67 & Peripheral lung infiltration & Squamous-cell carcinoma \\
12 & M & 62 & Pleural mass & Squamous-cell carcinoma \\
13 & M & 54 & Pleural mass & Malignant Schwannoma \\
14 & M & 39 & Pleural mass &
\end{tabular}

ANLL = acute non-lymphoid leukaemia

* With compression of vena cava. 
the pleura will be encountered and the location of the diaphragm can be determined clearly from the scans. Thus the needle can be positioned in the centre of the effusion without laceration of the lung, diaphragm, liver, or spleen. ${ }^{13}$ Ultrasonography should thus be the method of choice to guide thoracocentesis if the amount of pleural fluid is small or if the fluid is loculated.

Percutaneous biopsy and fine-needle aspiration biopsy of thoracic masses are also usually performed under fluoroscopic guidance. Fluoroscopic observation, however, may not allow a satisfactory view of a mass in two planes. It is also possible to damage interposed aerated lung, since the point of contact between the mass and the chest wall is difficult to ascertain. In addition to the exposure to radiation, it is often difficult to manipulate the needle with the radiological unit in position. Ultrasonography allows easy localisation of echogenic masses originating from the chest wall and the pleura and also from the lung and the mediastinum, provided that there is no interposition of aerated lung tissue. The direction of the needle and the depth of the mass at the puncture site can be accurately determined, so that puncturing other structures is easily avoided. Ultrasonography therefore seems to be the method of choice to guide puncture of these masses. ${ }^{14}$

In this series, the echo-guided puncture and aspiration of the intrathoracic tumours or effusions were performed as an initial diagnostic procedure in most cases. Patient 1 received local treatment by methotrexate instillation. Mediastinoscopy or mediastinotomy was required for further differentiation of the tumour origin in cases 3,4 , and 6 . Patient 8 refused further investigation, and patients 5 and 7 were excluded from surgical procedures because of caval compression. In each case the accuracy of the cytological diagnosis was proved by the follow-up study.

Ultrasound has also been used successfully for pericardiocentesis. ${ }^{15}$ It determines the best location and depth at which to position the needle. Furthermore, it allows continuous observation during the puncture. It should be obvious that this method is safer than the relatively blind procedures, still in common use, in which a needle is advanced through the pericardium by a subxiphoid or left parasternal approach.

Puncture of most thoracic lesions can be guided by a static B-scanner. If lesions are small, however, or if it is advantageous to control the puncture itself or the position of the surrounding structures during the puncture, then real-time sonographic control is necessary. In this series a real-time linear-array transducer was used. With this kind of instrument the needle tip cannot always be visualised, especially when the needle is small or when solid masses are punctured. This is because the needle is parallel to the ultrasonic beam. Punctures may also be guided by real-time sector scanners. Then the needle, penetrating the ultrasonic field obliquely, is more easily visualised. ${ }^{16-18}$ Finally, the needle may be guided by a single transducer with a central canal. The reflection from the tip of the needle is then monitored in A- or M-mode. ${ }^{19} 20$

In conclusion, effusions and solid masses in the thorax can easily be punctured under sonographic guidance, provided that they are in contact with the chest wall. Although a static B-scanner will usually be sufficient, if masses are small or if they are surrounded by vital structures that must be avoided punctures may be controlled by real-time scanners. Because even small lesions are accurately located, complications of puncture should be rare.

\section{References}

${ }^{1}$ Joyner CR Jr, Herman RJ, Reid JM. Reflected ultrasound in the detection and localisation of pleural effusion. JAMA 1967;200:399-402.

2 Sandweiss DA, Hanson JC, Gosink BB, Moser KM. Ultrasound in diagnosis localisation and treatment of loculated pleural empyema. Ann Intern Med 1975;82:50-3.

${ }^{3}$ Gryminski J, Krakowka P, Lypacewicz G. The diagnosis of pleural effusion by ultrasonic and radiologic techniques. Chest 1976;70:33-7.

4 Smulewicz JJ, Tafreshi M, Hassani N. Ultrasonic diagnosis and aspiration of loculated pleural effusion. Respiration 1977;34:36-8.

${ }^{5}$ Bontin B, Farisse P, Aimino R, Rosello R, Pietri H. Intérêt de l'échotomographie en pathologie pleuropulmonaire. Poumon Coeur 1976;32:9-19.

- Cunningham JJ. Gray scale echography of the lung and the pleural space. Cancer 1979;41:1329-39.

${ }^{7}$ Forsberg L, Tylen U. Ultrasound examination of lesions in the thorax. Acta Radiol (Diagn) 1980;21:375-8.

${ }^{8}$ Haller JO, Schneider M, Kassner EG, Friedman AP, Waldroup LD. Sonographic evaluation of the chest in infants and children. Am J Radiol 1980;134:1019-27.

' Feigenbaum H, Waldhausen JA, Hyde LP. The ultrasound diagnosis of pericardial effusion. JAMA 1965;191:711-4.

${ }^{10}$ Moss AJ, Bruhn F. The echocardiogram: an ultrasound technique for the detection of pericardial effusion. $N$ Engl J Med 1966;274:380-4.

"Soulen RL, Lapayowker MS, Gimenez JL. Echocardiography in the diagnosis of pericardial effusion. Radiology 1966;86:1047-51.

12 Goldberg BB, Ostrum BJ, Isard HJ. Ultrasonic determination of pericardial effusion. JAMA 1967;202:92730.

${ }^{13}$ Ravin CE. Thoracocentesis of loculated pleural effusions using gray scale ultrasonic guidance. Chest 1977;71:666-8. 
${ }^{14}$ Chandrasekhar AJ, Reynes CJ, Churchill RJ. Ultrasonically guided percutaneous biopsy of peripheral pulmonary masses. Chest 1976;70:627-30.

${ }^{15}$ Goldberg BB, Pollack HM. Ultrasonically guided pericardiocentesis. Am J Cardiol 1973;31:490-3.

${ }^{16}$ Lindgren PG. Ultrasonically guided punctures. Radiology 1980;137:235-7.

${ }^{17}$ Saitoh M, Watanabe H, Ohe H, Tanaka S, Itakura Y, Date S. Ultrasonic real-time guidance for percutaneous puncture. JCU 1979;7:269-72.
${ }^{18}$ Makunchi M, Bandai Y, Ito T, Watanabe G, Wada T, Abe $H$, Muroi $T$. Ultrasonically guided percutaneous transhepatic bile drainage. Radiology 1980;136:1659.

${ }^{19}$ Goldberg BB, Pollack HM. Ultrasonic aspiration transducer. Radiology 1972;102:187-9.

${ }^{20} \mathrm{Holm}$ HH, Kristensen JK, Rasmussen SN, et al. Ultrasound as a guide in percutaneous puncture technique. Ultrasonics 1972;10:83-6. 